\title{
Spin Coated Ag NPs SERS substrate: Role of electromagnetic and chemical enhancement in trace detection of methylene blue and congo red
}

Imran Shaikh

Savitribai Phule Pune University

Mohammed Abdul Haque

Savitribai Phule Pune University

Habib Pathan

Savitribai Phule Pune University

Shrikrishna Dattatraya Sartale ( $\nabla$ sdsartale@physics.unipune.ac.in )

Savitribai Phule Pune University https://orcid.org/0000-0002-6463-7655

\section{Research Article}

Keywords: SERS, trace detection, spin coating, Ag NPs, industrial pollutants

Posted Date: March 2nd, 2022

DOI: https://doi.org/10.21203/rs.3.rs-1381353/v1

License: (c) (i) This work is licensed under a Creative Commons Attribution 4.0 International License.

Read Full License 


\title{
Spin Coated Ag NPs SERS substrate: Role of electromagnetic and chemical enhancement in trace detection of methylene blue and congo red Imran Shaikh, Mohammed Abdul Haque, Habib Pathan and Shrikrishna Sartale* \\ Thin Films and Nanomaterials laboratory, Department of Physics, Savitribai Phule Pune University, Pune-411007, INDIA
}

\begin{abstract}
This work demonstrates a simple low cost and reproducible spin coating method followed by thermal reduction for the uniform growth of silver nanoparticles (AgNPs) as surface enhanced Raman scattering (SERS) substrate. In this work, cationic dye MB and anionic dye CR are selected as two different kinds of SERS probe molecules to investigate the differences between EME and CE mechanism. The results indicate that SERS spectra of MB molecule on AgNPs SERS substrate exhibit the shift in the Raman peak positions and high sensitivity (LOD 10 $0^{-10}$ M) with large $\mathrm{G}_{\mathrm{SERS}}\left(\sim 10^{9}\right)$ value, which can be attributed to the contribution from both EME and $\mathrm{CE}$ mechanism. It is found that $\mathrm{Cl}^{-}$halide ions present in the $\mathrm{MB}$ molecule are forming surface complex (AgNPs@ $\mathrm{Cl}^{-}$) on the surface of AgNPs SERS substrate which is confirmed by the XPS investigation, which might be responsible for the CE enhancement in MB molecule. Whereas Congo red being anionic dye showed no shift in Raman peak positions and less sensitivity $\left(\operatorname{LOD} \sim 10^{-8}\right)$ with GSERS $\left(\sim 10^{7}\right)$ value on AgNPs SERS substrate, which can be attributed to EME mechanism only. The obtained results suggest that $\mathrm{Cl}^{-}$halide ions present in the MB molecule plays important role by giving large SERS enhancement for MB molecule on AgNPs SERS substrate. It is expected that this work will provide insight into role of EME and $\mathrm{CE}$ mechanisms for cationic dye $\mathrm{MB}$ and anionic dye $\mathrm{CR}$.
\end{abstract}

KEYWORDS: SERS; trace detection; spin coating; Ag NPs; industrial pollutants.

*Corresponding author:

E-mail: sdsartale@physics.unipune.ac.in 


\section{INTRODUCTION}

Observation of intense Raman spectrum of pyridine molecule adsorbed on the roughened Ag electrode substrate by Fleishmann et al. [1] opened up new means of exploring Raman spectroscopy in terms of surface enhanced Raman scattering (SERS). Right from the discovery of SERS technique up till now various groups have made lots of efforts so as to give an insight of SERS technique and to develop SERS substrate with maximum enhancement [2-9]. SERS enhances the Raman signal up to $10^{13}-10^{15}$ times which makes SERS as an ultrahigh sensitive technique for the trace detection of various molecules [4, 7, 8, 10-12]. Electromagnetic field enhancement (EME) and chemical enhancement (CE) are considered to be the main mechanisms responsible for such huge enhancement in the Raman scattering [7, 10, 13]. Amongst the duo, EME has always been the centre of interest because of its major contribution $\left(10^{11}-10^{12}\right)$ as compared to CE $\left(10^{2}-10^{3}\right)$ in the SERS $[11,12]$. EME arises due to the localized surface plasmon resonance (LSPR) occurring in the plasmonic metal nanoparticles (NPs) such as silver (Ag), gold $(\mathrm{Au})$, copper $(\mathrm{Cu})$, etc. LSPR occurs due to confinement of plasmons (collective oscillations of the electron gas against the ion core in the metal) near the surface under light irradiation of the plasmonic metal NPs, which facilitates huge local electric field near the edges or in the vicinity [14]. This generated huge local electric field then interacts with the molecule adsorbed in the proximity of plasmonic metal NPs and enhances its Raman scattering. Size, shape, inter-particle distance and incident laser's wavelength and intensity are crucial parameters deciding the strength of EME. Plasmonic metal NPs based SERS substrates gained utmost priority due to their high sensitivity and reproducibility of the SERS signal [3]. Many studies show that LSPR peak coinciding with the wavelength of incident laser produces huge local electric field in the proximity of plasmonic metal NPs [14-16]. Therefore, optimizing the size, shape, and interparticle distance of appropriate plasmonic metal NPs with suitable coinciding excitation source greatly enhances SERS signal. Sensitivity of the SERS substrate depends on so called "hot spot" on SERS substrate where plasmonic metal NPs are arranged much closure (typically 1-10 nm) to each other $[3,17]$. Hot spot generates huge local electric field in the proximity of plasmonic metal NPs which enhances the SERS signal and hence detection limit of the probe molecule increases. On the other hand another important parameter reproducibility of the SERS signal of the probe molecule depends on degree of uniform distribution of plasmonic metal NPs. 
Interaction of probe molecule with the metal NPs surface facilitates transfer and/or exchange/sharing of electron between them which leads to the CE mechanism [13]. EME mechanism is not selective to the probe molecule; however strength of CE depends upon the type of interaction between the probe molecule and the metal NPs surface. Naturally the intrinsic structure of the probe molecule and the morphology of metal NPs surface decides the type of interaction and hence the strength of CE. Qualitatively CE can be investigated by looking at the shift $\left(5-30 \mathrm{~cm}^{-1}\right)$ in the Raman peak positions [18]. Jiang et al. studied the difference between EME and CE mechanisms contribution for 4-mercaptobenzoic acid (4-MBA) molecule when adsorbed on two different SERS substrate namely semiconductor $\mathrm{TiO}_{2}$ SERS substrate and Ag NPs SERS substrate [19]. They found evident difference in the spectral behavior, limit of detection (LOD) and $\mathrm{G}_{\mathrm{SERS}}$ values for 4-MBA molecule adsorbed on $\mathrm{TiO}_{2}$ SERS substrate and Ag NPs SERS substrate and attributed to the essential differences between EME and CE mechanisms. Kneipp et al. reported that for same average plasmonic field enhancement dyes having small structural variations give the difference in the contributions of the CE to the total SERS enhancement [20]. It is also reported that adsorption of cationic dye (dye whose components dissociate into positively charged ions in an aqueous solution) on Ag-halide $\left(\mathrm{Cl}^{-}, \mathrm{Br}^{-}\right.$ and $\mathrm{I}^{-}$) surface complex facilitate charge transfer leading to CE mechanism [21-23]. Stefancu et al. shown that AgNPs@ $\mathrm{Cl}^{-}$surface complex favors the adsorption of cationic dyes to the Ag NPs with strongest affinity and AgNPs@ $\mathrm{Mg}^{2+}$ surface complex favors the adsorption of anionic dye (dye whose components dissociates into negatively charged ions in an aqueous solution) to the Ag NPs with strongest affinity [23]. They also showed that anionic dye and cationic dye do not interact strongly with AgNPs@ $\mathrm{Cl}^{-}$and AgNPs@ $\mathrm{Mg}^{2+}$ surface complex, respectively. Hence both the mechanisms combinely play vital role to boost total SERS enhancement. Therefore in order to get highly sensitive and reproducible SERS substrate plasmonic metal NPs must be close to each other and uniformly distributed all over the large area support and probe molecule must have greater affinity to the metal NPs surface.

Textile industrial wastages are considered to be one of the major causes of water pollution. Textile industry uses various types of dyes for coloring the fabrics, and many of them are toxic, mutagenic and carcinogenic in nature [24]. Therefore not only it makes bad impact on human beings but also on the ecosystem of the earth. Textile colorant released in the water harms 
the aquatic life making the adverse effect on the food chain. Therefore it is important to have a technique like SERS which can detect toxic dyes in trace amount.

Amongst the plasmonic metal NPs, Ag NPs gained tremendous popularity as SERS substrate due to its narrow plasmonic band width in the visible region. The resonance quality of the plasmonic metal NPs depends on the width of plasmon resonance which can be given by the term ' $\Upsilon\left(\varepsilon_{b}+3\right)$ ', where $\Upsilon$ is the electronic scattering rate and $\varepsilon_{\mathrm{b}}$ is the contribution of inter-band transitions to the dielectric function [10]. Lower the plasmonic width, higher is the quality of the resonance and vice versa. The values of $\Upsilon$ and $\varepsilon_{b}$ for $\mathrm{Ag}$ NPs in the visible region are comparatively lower than $\mathrm{Au}$ and $\mathrm{Cu}[10]$, and hence $\mathrm{Ag}$ NPs are first choice to be used as a highly efficient SERS substrate. Many techniques have been adopted to grow large scale highly sensitive and reproducible Ag NPs based SERS substrates such as low-energy ion beam sputtering, pulsed laser deposition, electron-beam lithography, magnetron sputtering, electrodeposition, etc.[25-31], but these methods are sophisticated and expensive. In this work we have grown Ag NPs by simple and inexpensive spin coating method. The advantage of spin coating method is that one can easily tune the size, shape and coverage (separation) of the grown metal NPs just by varying growth parameters (concentration of the precursors, rpm, ramp, drop volume, etc.) of spin coating method [32-35].

In the present work Ag NPs of different size and coverage were grown on glass substrate $\left(1 \times 1 \mathrm{~cm}^{2}\right)$ by varying rpm of spin coating method to be used as a SERS substrate. To the best of our knowledge there are no reports on Ag NPs grown by spin coating followed by thermal reduction for the SERS application. Furthermore, present work sheds light on the contribution of CE which plays crucial role in deciding overall SERS enhancement and low detection limit (LOD) of the probe molecules. We have chosen two industrial pollutants namely methylene blue (MB) and congo red (CR) which are cationic [36] and anionic [37] in nature in order to investigate their SERS activity on Ag NPs grown by spin coating followed by thermal reduction. Theoretical modelling in order to calculate the spatial local electric field in the proximity of Ag NPs grown at different rpm by spin coating followed by thermal reduction was performed by the commercial COMSOL multiphysics software. Theoretically and experimentally obtained enhancement factors distinguish the contribution of EME and CE mechanism in case of MB and CR. 


\section{EXPERIMENTAL}

Experimental details to grow Ag NPs by spin coating followed by thermal reduction are discussed in our previous work [32, 38] and shown systematically in Figure 1. In a typical experiment to grow $\mathrm{Ag} \mathrm{NPs}, 50 \mu \mathrm{l}$ of $0.01 \mathrm{M} \mathrm{AgNO}_{3}$ ethanolic solution was dropped on glass substrate $\left(\approx 6 \mathrm{~cm}^{2}\right)$ and then spun for $30 \mathrm{~s}$ at different rpm (1000, 3000 and 5000) achieved with $200 \mathrm{rpm} / \mathrm{s} \mathrm{ramp} \mathrm{in} \mathrm{order} \mathrm{to} \mathrm{get} \mathrm{uniform} \mathrm{coverage} \mathrm{of} \mathrm{AgNO}_{3}$ on the glass substrate. At the end $\mathrm{AgNO}_{3}$ coated glass substrates were kept in the tube furnace for the thermal reduction at $350{ }^{\circ} \mathrm{C}$ achieved with $3{ }^{\circ} \mathrm{C} / \mathrm{min}$ heating rate for $1 \mathrm{~h}$ in $\mathrm{H}_{2}\left(3 \% \mathrm{H}_{2}\right.$ in $\left.\mathrm{Ar}\right)$ environment to convert $\mathrm{AgNO}_{3}$ into Ag NPs. Ag NPs grown at 1000, 3000 and $5000 \mathrm{rpm}$ by spin coating followed by thermal reduction named as AgT1k, AgT3k and AgT5k, respectively. Visible changes can be seen after and before the deposition of Ag NPs on glass substrate (See supplementary figure S1). After deposition glass substrates color changes to metallic grey indicating growth of Ag NPs on the glass substrate (See supplementary figure S1).

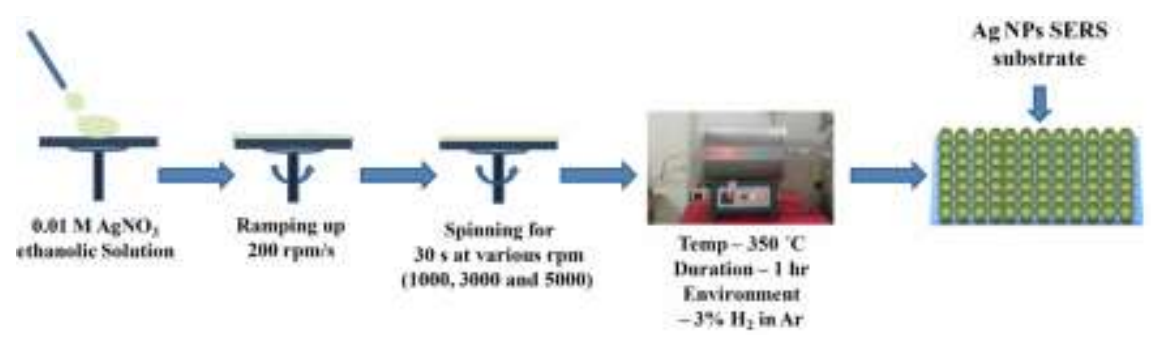

Figure 1: Schematic of Ag NPs growth by spin coating followed by thermal reduction.

In contrast to our earlier work [32] we have used piranha solution to clean the glass substrates $\left(\approx 6 \mathrm{~cm}^{2}\right)$ and used $350{ }^{\circ} \mathrm{C}$ as a thermal reduction temperature in order to grow Ag NPs suitable for SERS application. Glass substrates were immersed in mixed solution of $\mathrm{H}_{2} \mathrm{SO}_{4}$ (24 $\mathrm{ml})$ and $\mathrm{H}_{2} \mathrm{O}_{2}(6 \mathrm{ml})$ in $4: 1$ ratio for $3 \mathrm{~h}$. It is well known that substrate cleaned in piranha solution becomes hydrophilic due to the adsorption of hydroxyl groups onto the substrate [39, 40]. For lower rpm (< 1000) we observed that large sized Ag NPs merged with each other, whereas for higher rpm (>5000) sparsely distributed tiny Ag NPs were observed. For SERS application neither merged large nor tiny NPs with large separation are considered to be useful, hence we studied effect of rpm between 1000 to $5000 \mathrm{rpm}$. 
JASCO UV-Vis-NIR spectrophotometer model V-670 was used to investigate the optical properties of the grown Ag NPs. Industrial pollutant MB was used as a probe molecule to optimize the spin coating parameters. The inVia Renishaw micro-Raman spectrophotometer with an excitation source of $532 \mathrm{~nm}$ having power $0.01 \%$ of $60 \mathrm{~mW}$, spot size of $15 \mu \mathrm{m}$ and 50x long working distance objective was used for Raman measurements. For SERS measurements $10 \mu 1$ aqueous solution of probe molecule (volume of the probe molecule for all concentrations of MB or CR was kept constant) was drop casted on Ag NPs grown on glass substrate with the help of micro-pipette and then dried in air. Surface topology and roughness of Ag NPs grown at various rpm (1000, 3000 and $5000 \mathrm{rpm})$ by spin coating method were investigated using commercially available Multimode 8.0, Bruker, atomic force microscope (AFM) with scan assist mode. Surface morphology of optimized Ag NPs SERS substrate studied using Jeol JSM-6360A instrument having operating voltage $20 \mathrm{kV}$. PHI Versaprobe III having $\mathrm{Al} \mathrm{K \alpha} \mathrm{X}$-rays was used for X-ray photoelectron spectroscopy (XPS) investigation. The distributions of spatial electric field in the proximity of Ag NPs were investigated with the help of RF module of commercial COMSOL multiphysics software.

\section{RESULTS AND DISCUSSION}

The plasmonic metal NPs ( $\mathrm{Au}, \mathrm{Ag}, \mathrm{Cu}$, etc.) show LSPR which can be easily detected by UV-visible absorption spectroscopy. Variations in the peak position and intensity of the LSPR indicate the changes in the size, shape and coverage of the plasmonic metal NPs. Figure 2 shows UV-vis absorption spectra of AgT1k, AgT3k and AgT5k. The absorption spectra of Ag NPs show blue shift in LSPR peak positions (441 to $428 \mathrm{~nm}$ ) and decrease in the peak intensity with increase in rpm (1000 to 5000) suggesting decrease in the size and the coverage of Ag NPs. Obtained LSPR peak for Ag NPs grown at various rpm indicate narrow size distribution, suggesting major contributions of small sized spherical Ag NPs. From SEM image of AgT1k (see supplementary information figure S2), it is evident that Ag NPs are uniformly grown all over the glass substrate. Most of the grown Ag NPs are of spherical in shape with very few bigger sized spherical Ag NPs giving rise to the narrow size distribution. 


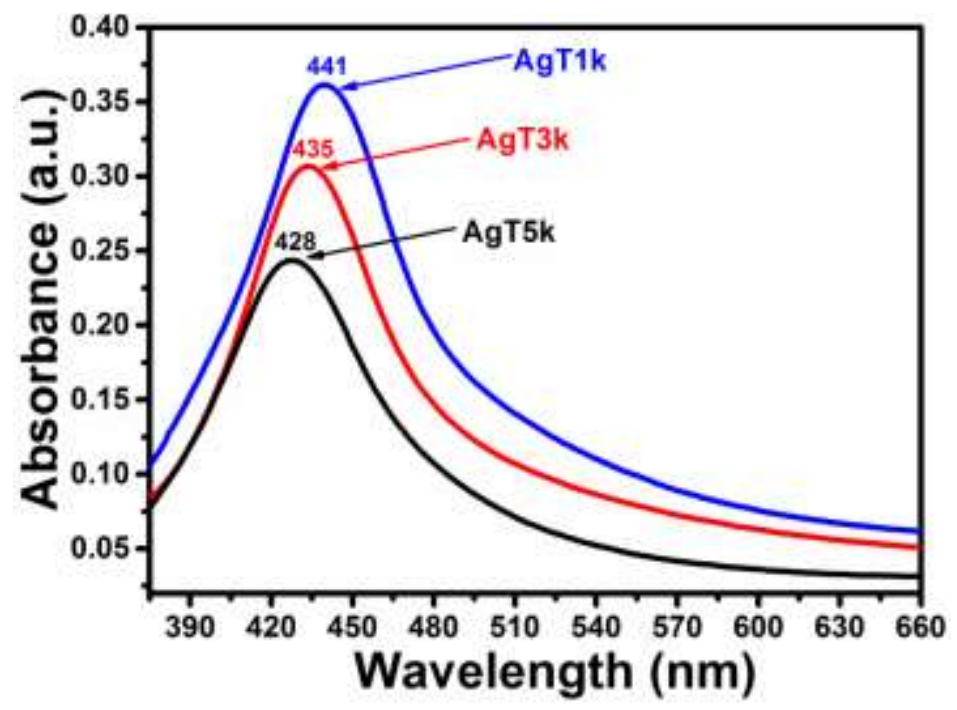

Figure 2: UV-vis absorption spectra of AgT1k, AgT3k and AgT5k.

The growth process of Ag NPs can be explained as follows; after dropping the solution (solute $\left(\mathrm{AgNO}_{3}\right)+$ solvent (ethanol)) onto the substrate acceleration (ramp) is given to achieve desired rpm. At certain point of time formation of uniform film of the solution takes place on the substrate due to the balance set up between shear and centrifugal forces. Slowly uniform film thickness starts decreasing due to dominance of evaporation. Evaporation leads to increase in the solute concentration which in turn supersaturates the solvent triggering nuclei formation. The nuclei then further start growing in the form of crystal leading to decrease in the concentration of the solution preventing further nuclei formation [34, 35, 41]. Uniformly coated $\mathrm{AgNO}_{3}$ nanocrystals on the substrate placed in the tube furnace at $350{ }^{\circ} \mathrm{C}$ in $\mathrm{H}_{2}$ environment for $1 \mathrm{hr}$ to reduce $\mathrm{Ag}^{+}$ions from $\mathrm{AgNO}_{3}$ into the $\mathrm{Ag}^{0}$ resulting into growth of $\mathrm{Ag}$ NPs. It is well established that for a constant evaporation rate, amount of spin coated material to be deposited on the flat substrate is the function of viscosity of precursor solution $(\eta)$, initial concentration of active component in the dropped solution $\left(\mathrm{C}_{0}\right)$ and rotational frequency $(\omega)$. For fixed $\mathrm{C}_{0}$ and $\eta$ the material to be spin coated on the substrate is proportional to $\omega^{-\mathrm{x}}$, where $\mathrm{x}(0 \leq \mathrm{x} \leq 1)$ is the function of type of fluid and ramp [34, 35, 42]. Therefore with increase in rpm amount of spin coated $\mathrm{AgNO}_{3}$ and hence the size of the grown $\mathrm{Ag}$ NPs decreases.

Figure 3 shows SERS spectra of MB $\left(10^{-5} \mathrm{M}\right)$ adsorbed on AgT1k, AgT3k and AgT5k. All obtained Raman peaks matched well with the reported literature (see supporting information Table S1) with shift in peak positions suggesting chemisorption of MB molecule on the grown 
AgNPs [43]. In Figure 3 a) $\mathrm{C}-\mathrm{C}$ ring stretching mode of $\mathrm{MB}$ molecule gives rise to the most intense peak around $1624 \mathrm{~cm}^{-1}$. The peaks at about 447 and $499 \mathrm{~cm}^{-1}$ assigned to the C-N-C skeletal deformation mode. Skeletal deformation of C-S-C gives rise to the weak peak at $594 \mathrm{~cm}^{-}$ 1. Out of plane and in plane bending of $\mathrm{C}-\mathrm{H}$ modes give rise to the medium and weak intense peaks around 669, 769, 858, 899, 950, 1038, 1071 and $1153 \mathrm{~cm}^{-1}$. Similarly the medium and strong intense peaks around 1300 and $1395 \mathrm{~cm}^{-1}$ arise due to in plane ring deformation of the C$\mathrm{H}$ mode. In plane ring deformation of $\mathrm{C}-\mathrm{N}$ mode gives rise to a weak intense peak around 1179 $\mathrm{cm}^{-1}$, also peaks around 1443 and $1468 \mathrm{~cm}^{-1}$ can be assigned to the asymmetric stretching of the C-N mode [44-46]. It is also evident that the SERS activity decreases with increase in the rpm. It is well known that closer the LSPR peak of Ag NPs to the excitation source maximum will be the local electric field in the proximity of Ag NPs resulting in large SERS enhancement. Therefore, AgT1k gives largest SERS enhancement and it is used for further studies.
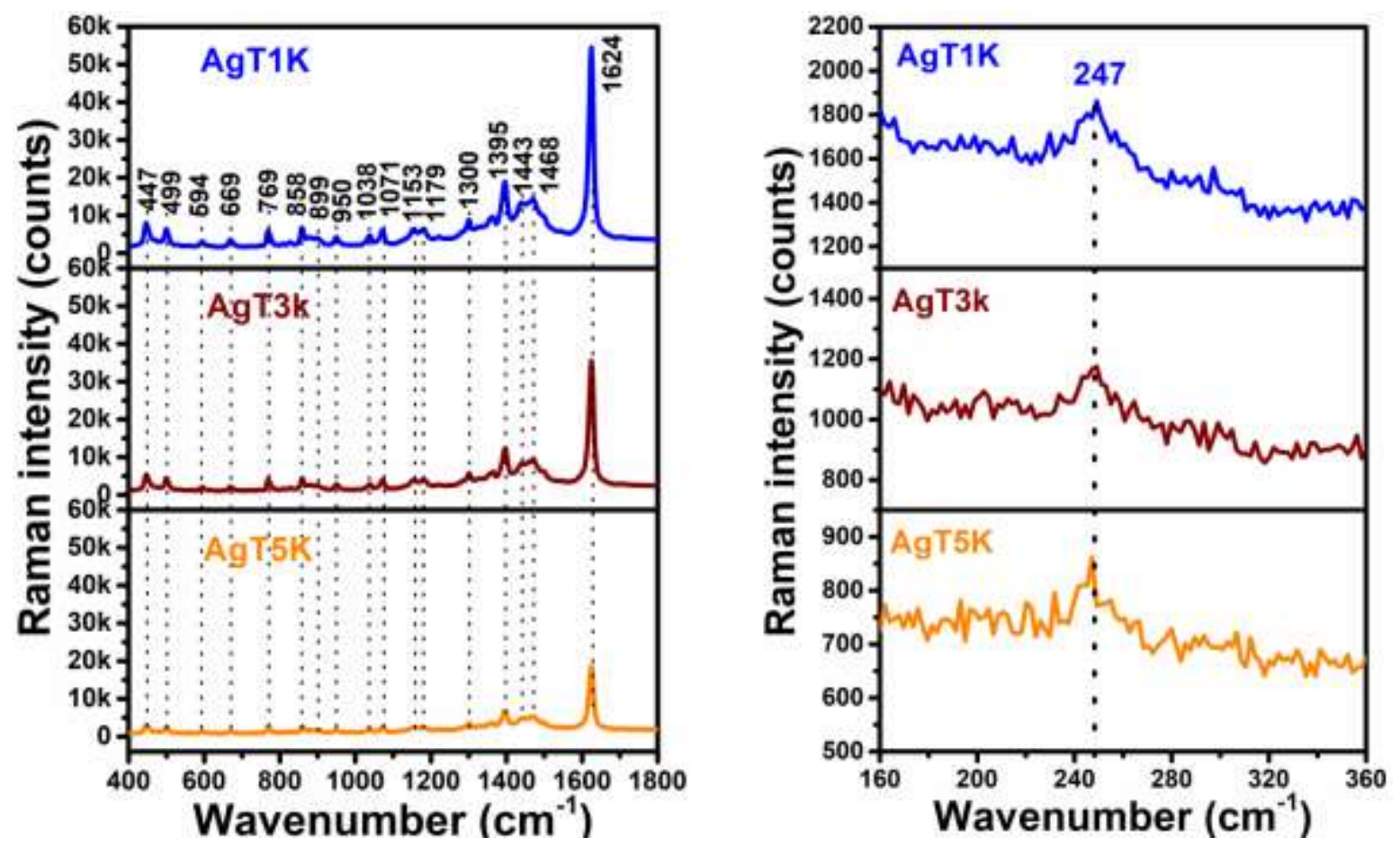

Figure 3: SERS spectra of MB $\left(10^{-5} \mathrm{M}\right)$ on AgT1k, AgT3k and AgNPs-T5k a) 400 to $1800 \mathrm{~cm}^{-1}$ and b) 160 to $360 \mathrm{~cm}^{-1}$ wavenumbers.

Careful examination of SERS spectra of MB molecule at lower wavenumbers (Figure 3 b) shows presence of a very weak Raman peak at $247 \mathrm{~cm}^{-1}$ which can be assigned to the stretching mode of $\mathrm{AgCl}$ [47]. Presence of $\mathrm{AgCl}$ Raman peak in the SERS spectra of MB 
molecule suggests the formation of AgNPs@ $\mathrm{Cl}^{-}$surface complex on the surface of $\mathrm{Ag}$ NPs SERS substrate. $\mathrm{Cl}^{-}$halide ion is present in the structure of $\mathrm{MB}$ molecule. It is well known that halide ions show strong affinity to the Ag nanoparticles. Therefore there is a possibility that when aqueous solution of MB molecule drop casted on to the Ag NPs and dried in the air prior to SERS measurements, $\mathrm{Cl}^{-}$ions present in the MB molecule may get adsorbed on to the Ag NPs surface and form AgNPs@ $\mathrm{Cl}^{-}$surface complex due to its high affinity to Ag NPs [23]. AgNPs@ $\mathrm{Cl}^{-}$surface complex favors the charge transfer between cationic MB dye and Ag NPs surface [21-23]. Further the intensity of Raman peak due to $\mathrm{AgCl}$ decreases with decrease in concentration of $\mathrm{MB}$ endorsing AgNPs@ $\mathrm{Cl}^{-}$surface complex formation (See supplementary figure S3). Hence shift in the peak positions and presence of the Raman peak at $247 \mathrm{~cm}^{-1}$ in SERS spectra of MB molecule confirms the chemisorption of MB molecule on Ag NPs surface.

Figure 4 shows AFM images of AgT1k, AgT3k and AgT5k. From AFM images it is observed that the Ag NPs are uniformly grown and their size decreases with increase in rpm, in line with optical absorption results [32]. For fixed viscosity and concentration of the precursor solution the amount of material spin coated on the substrate is inversely proportional to the square root of the rotational frequency $(\omega)[48,49]$. Since the spin coated material is less at higher rpm decrease in the size of grown Ag NPs is expected. The size and separation between the Ag NPs taken from AFM images for simulation were average ones. We have used the RF module of the COMSOL multiphysics software in order to measure the spatial electric field distribution around the isolated and dimer Ag NPs (Fig. 4). For simulation we have set incident electric field ( $E_{\text {incident }}$ ) at $1 \mathrm{~V} / \mathrm{m}$ with incident laser of wavelength $532 \mathrm{~nm}$. The necessary optical constants of Ag required in simulation were taken from Johnson and Christy's data of optical properties of metals [50]. Size and separation used in the COMSOL multiphysics simulation modeling and obtained local electric field ( $\mathrm{E}_{\text {local }}$ ) for isolated and dimer Ag NPs grown at various rpm are summarized in Table 1. 
Table 1 COMSOL multiphysics simulation modeling parameters obtained from AFM images and calculated local electric field ( $\left.E_{\text {local }}\right)$ for isolated and dimer Ag NPs grown at various rpm.

\begin{tabular}{cccccc}
\hline rpm & $\begin{array}{c}\text { Size } \\
(\mathbf{n m})\end{array}$ & $\begin{array}{c}\text { Separation } \\
(\mathbf{n m})\end{array}$ & $\begin{array}{c}\mathbf{E}_{\text {local }} \text { of isolated } \\
\mathbf{A g ~ N P}(\mathbf{V} / \mathbf{m})\end{array}$ & $\begin{array}{c}\mathbf{E}_{\text {local }} \text { of dimer } \\
\mathbf{A g ~ N P s}(\mathbf{V} / \mathbf{m})\end{array}$ & $\begin{array}{c}\text { SERS EF } \\
\left(\mathbf{e m}_{\text {GsERs }}\right)\end{array}$ \\
\hline 1000 & 100 & 3 & 9.98 & 81.2 & $4.3 \times 10^{7}$ \\
3000 & 67 & 3 & 6.53 & 36.6 & $1.8 \times 10^{6}$ \\
5000 & 58 & 4 & 5.41 & 22.1 & $2.4 \times 10^{5}$ \\
\hline
\end{tabular}

$\mathrm{E}_{\text {local }}$ around the isolated and in between Ag NPs found to decrease as the size of Ag NPs gets decreased. Maximum $E_{\text {local }}$ enhancement for the dimer AgT1k is found to be $E_{\text {local }}=81.2 \mathrm{~V} /$ $\mathrm{m}$. Therefore, for $\mathrm{E}_{\text {incident }}=1 \mathrm{~V} / \mathrm{m}$ (set in the $\mathrm{COMSOL}$ simulation) theoretical electromagnetic enhancement factor $\left({ }^{\mathrm{em}} \mathrm{G}_{\text {SERS }}\right)$ can be written as ${ }^{\mathrm{em}} \mathrm{G}_{\text {SERS }}=\left[\mathrm{E}_{\text {local }}{ }^{4}[8,51]\right.$. For maximum $\mathrm{E}_{\text {local }}$, ${ }^{\mathrm{em}} \mathrm{G}_{\text {SERS }}$ of AgT1k approaches to $4.3 \times 10^{7}$. Thus AgT1k found to be optimized SERS substrate demonstrating maximum SERS enhancement and used for further studies. Calculated ${ }^{\mathrm{em}} \mathrm{G}_{\text {SERS }}$ value gives pure contribution of EME because no chemical interaction between the molecule and Ag NPs is considered. 

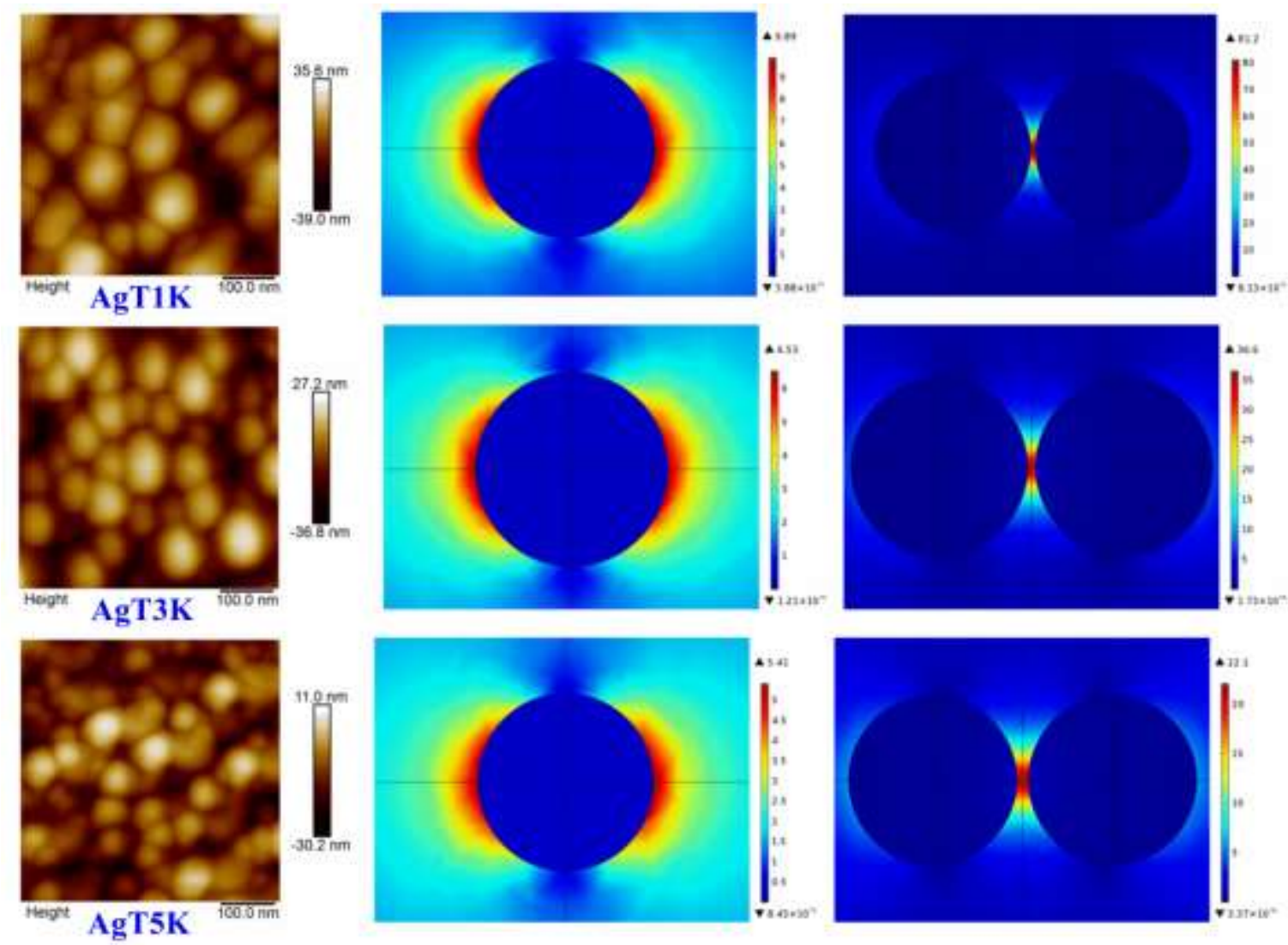

Figure 4: AFM images and spatial electric field distribution of the isolated and dimer Ag NPs of AgT1k, AgT3k and AgT5k.

Figure 5a shows sensitivity measurement of the AgT1k. It can be observed that as MB concentration decreases intensities of characteristic Raman peaks of MB molecule also decreases. We have checked the sensitivity of our SERS substrate up to $10^{-11} \mathrm{M}$ concentration of MB. However, for $10^{-11} \mathrm{M}$ concentration no peaks of MB were clearly distinguishable only a noisy peak at around $1624 \mathrm{~cm}^{-1}$ was detected therefore we have constrained ourselves only up to $10^{-10} \mathrm{M}$ concentration where all the MB peaks are clearly distinguishable. Calculation for SERS 

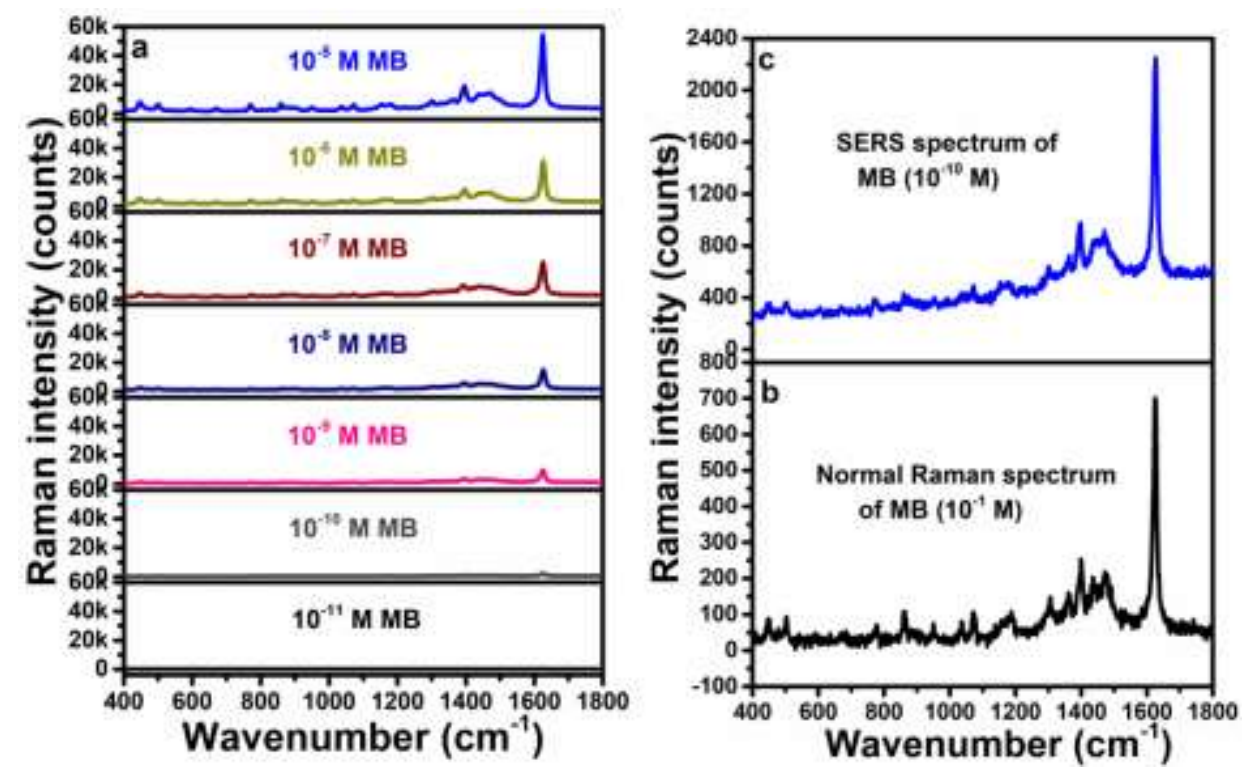

Figure 5: a) SERS spectra of MB having various molar concentrations $\left(10^{-5}-10^{-11} \mathrm{M}\right)$ on AgT1k SERS substrate, b) Normal Raman spectrum of $10^{-1}$ M MB on glass substrate and c) SERS spectrum of $10^{-10} \mathrm{M}$ MB on AgT1k SERS substrate.

enhancement factor was carried out for normal Raman spectra of MB having $0.1 \mathrm{M}$ concentration on glass substrate and SERS spectrum of MB having $10^{-10} \mathrm{M}$ concentration on AgT1k SERS substrate. SERS enhancement factor (GSER) equation (see supplementary information) can be written as below $[16,52]$,

$$
G_{S E R S} \sim \frac{M_{\text {bulk }}}{M_{\text {SERS }}} \times \frac{I_{S E R S}}{I_{\text {bulk }}}
$$

Where $M_{\text {bulk }}$ and $M_{S E R S}$ denote the number of MB molecules adsorbed onto glass and AgT1k. $I_{S E R S}$ and $I_{\text {bulk }}$ are the SERS and normal Raman peak intensities of the most intense peak of MB molecule, respectively. Normal Raman intensity of the MB (0.1 M) and SERS intensity of MB $\left(10^{-10} \mathrm{M}\right)$ at $1624 \mathrm{~cm}^{-1}$ were found to be 700 and 2250 counts (Fig. $5 \mathrm{~b}$ and c) and the SERS enhancement factor was found to be $\mathrm{G}_{\text {SERS }} \sim 3.2 \times 10^{9}$. Therefore the rest contribution $\left(\sim 10^{2}\right)$ is most probably from the CE mechanism which is confirmed by the observed shift in the peak position and presence of $247 \mathrm{~cm}^{-1}$ Raman peak of AgNPs@ $\mathrm{Cl}^{-}$surface complex in MB SERS spectra [53]. It is quite difficult to compare the obtained GSERS value with the available literature due to the fact that different MB concentrations, laser sources with different powers were used in the literature to calculate the GSERS values $[16,43,54]$. However it is noteworthy with low laser 
power $(60 \mu \mathrm{W})$, the AgT1k SERS substrate shows excellent sensitivity $\left(10^{-10} \mathrm{M}\right)$ and SERS enhancement $\left(\mathrm{G}_{\mathrm{SERS}} \sim 3.2 \times 10^{9}\right)$ factor as compared with the literature.

The relative standard deviation (RSD) was calculated for AgT1k in order to demonstrate the homogeneity of the grown Ag NPs SERS substrate. We have recorded the SERS spectra for $10^{-5} \mathrm{M} \mathrm{MB}$ at 14 different spots on the entire area of the AgT1k SERS substrate $\left(\approx 6 \mathrm{~cm}^{2}\right)$ (figure 6a). It is noteworthy that no change in the characteristics peaks of the MB molecule at the different spots with only minute variations in the intensity was observed. RSD in the SERS spectra at $1624 \mathrm{~cm}^{-1}$ at 14 different spots was found to be $6.72 \%$. The found RSD value is under the acceptable value mentioned in scientific standards for practical use of SERS substrate [55].
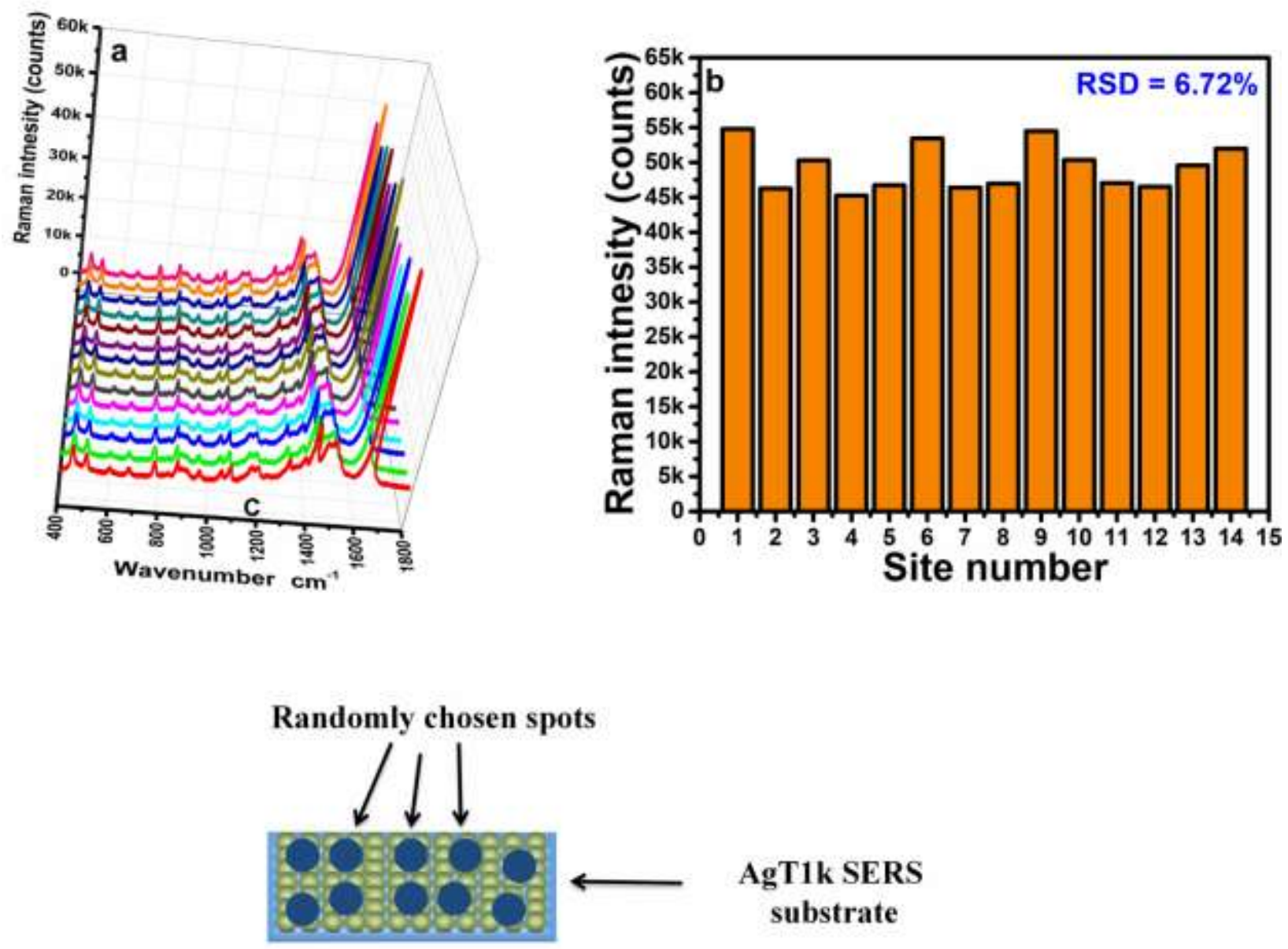

Figure 6: a) SERS spectra and b) SERS intensities of most intense MB molecules characteristics peak $\left(1624 \mathrm{~cm}^{-1}\right)$ recorded at randomly chosen 14 different spots on AgT1k SERS substrate and c) schematic of reproducibility measurement. 
Similarly, to check the substrate to substrate reproducibility of SERS signal of MB, we have made 8 different batches of the substrate for which RSD was found to be $10.93 \%$ (Figure 7). The found RSD is under the acceptable scientific standards (> 20\%) for SERS substrate [56]. Obtained results show that AgT1k SERS substrate shows excellent SERS enhancement factor with good reproducibility of the SERS signal all over the SERS substrate.
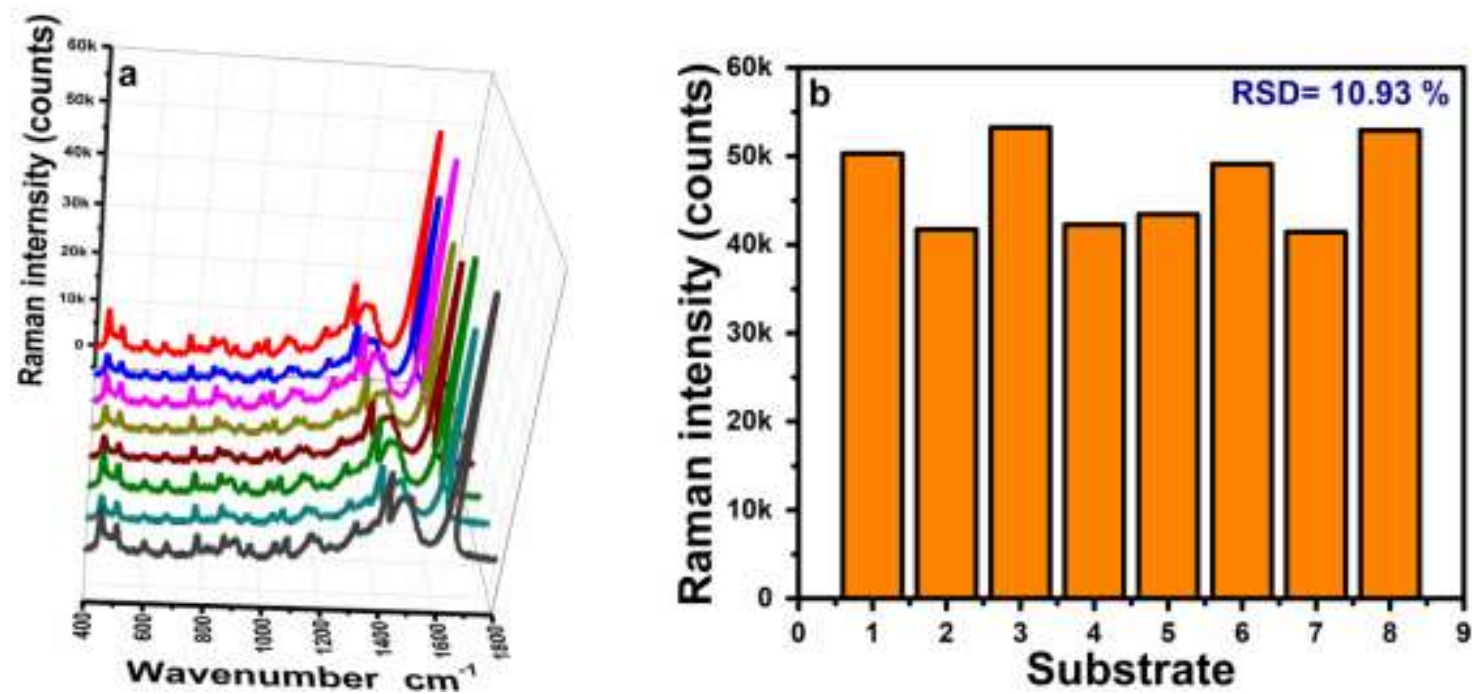

Figure 7: a) SERS spectra of $10^{-5} \mathrm{M} M B$ and b) SERS intensities of most intense MB molecules characteristics peak $\left(1624 \mathrm{~cm}^{-1}\right)$ for 8 different batched of AgT1k SERS substrate.

We have also investigated the reusability property of AgT1k SERS substrate. AgT1k SERS substrate was washed in double distilled water after detection and then again aqueous solution of MB adsorbed on it for the next cycle of detection. Figure 8 shows the reusability SERS spectra of AgT1k SERS substrate for MB having concentration of $10^{-5} \mathrm{M}$. Although with minimal intensity characterisitc Raman peak of MB molecule were observed even after washing indicating MB molecules were not completely rinsed off from the substrate. Obtained results confirm the chemosorption of MB molecule on AgT1k SERS substrate. 


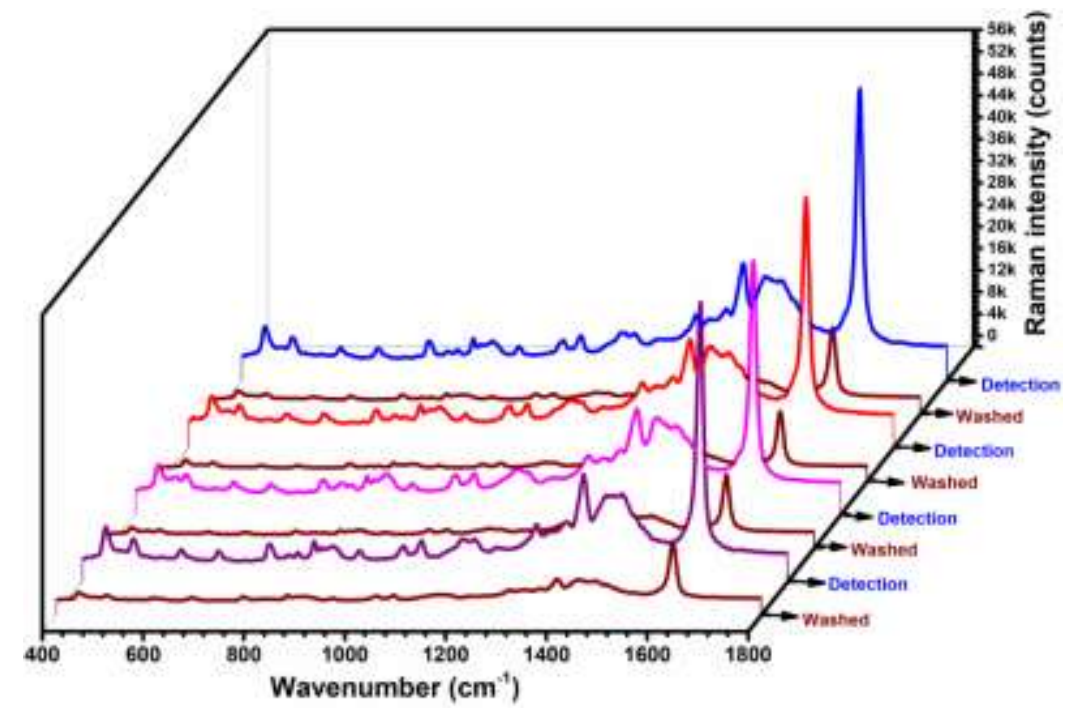

Figure 8: Reusability studies $10^{-5} \mathrm{M}$ MB on AgT1k SERS substrate.

Further we have detected another industrial pollutant namely CR in order to show the universality of our AgT1k SERS substrate. We have also carried out the sensitivity measurement up to $10^{-9} \mathrm{M}$ concentration of $\mathrm{CR}$. Figure 9a shows SERS spectra of $\mathrm{CR}$ at various concentrations $\left(10^{-5}-10^{-9} \mathrm{M}\right)$. All the obtained characteristics Raman peaks of CR matched very well with the literature $[57,58]$. There is no drastic shift in characteristic peaks observed in normal Raman and SERS spectra of CR (see supplementary information Table S4) indicating no chemisorption of CR molecules on AgT1k SERS substrate.

SERS spectrum of CR having $10^{-8} \mathrm{M}$ concentration was used to calculate GSERS. SERS enhancement measurement was carried out for the most intense peak of CR observed at 1376 $\mathrm{cm}^{-1}$ which arises due to the $\mathrm{N}=\mathrm{N}$ stretching. The normal Raman intensity of $\mathrm{CR}\left(10^{-1} \mathrm{M}\right)$ on glass substrate and SERS intensity of CR $\left(10^{-8} \mathrm{M}\right)$ on AgT1k SERS substrate for most intense peak were found to be 1375 and 4671 counts (Figure $9 \mathrm{~b}$ and c). From equation 1 the GSERS value for $M_{\text {bulk }}\left(10^{-1} M\right)$ and $M_{S E R S}\left(10^{-8} M\right)$ was found to be $\sim 3.4 \times 10^{7}$. Obtained experimental value is in good agreement with the theoretically calculated SERS enhancement factor value endorsing only EME contribution in SERS enhancement. 

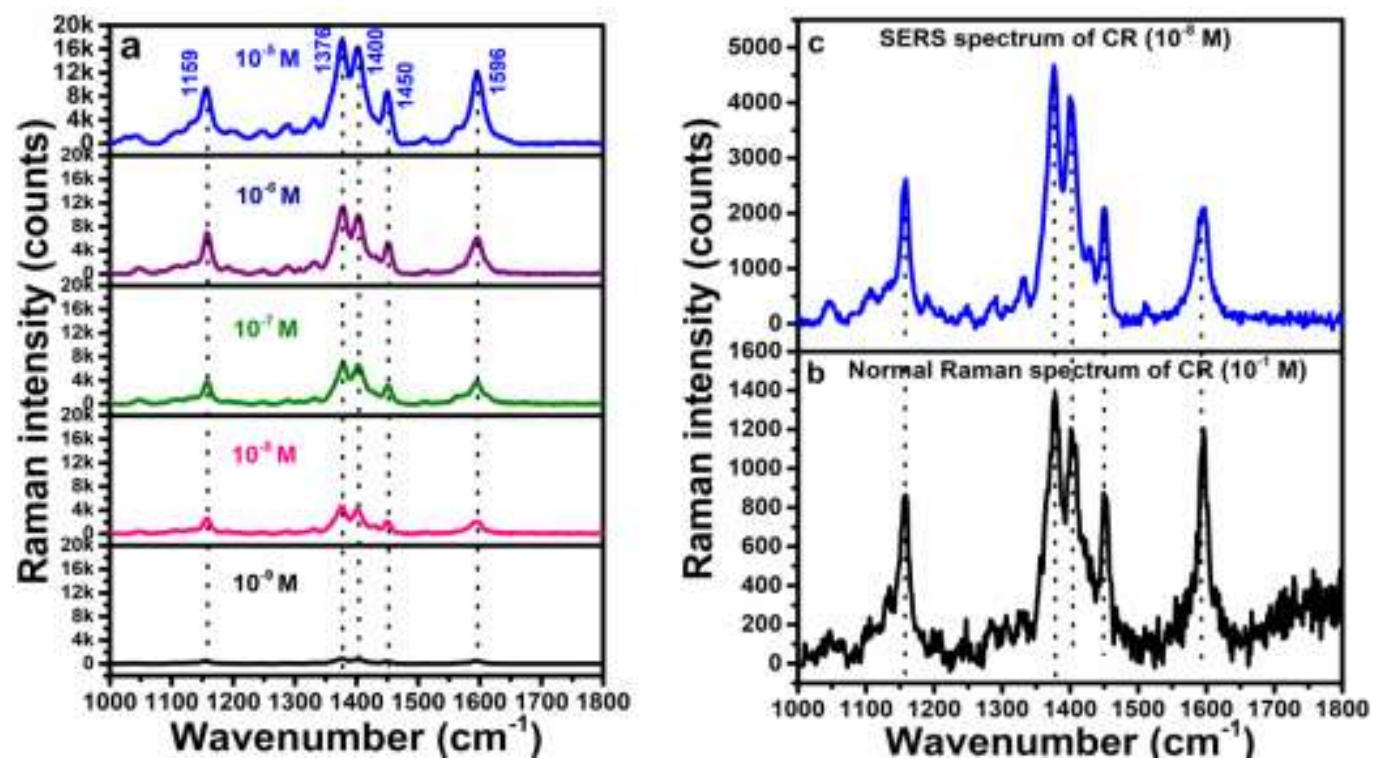

Figure 9: a) SERS spectra of CR having various molar concentrations $\left(10^{-5}-10^{-9} \mathrm{M}\right)$ on $\mathrm{AgT} 1 \mathrm{k}$ SERS substrate b) Normal Raman spectrum of $10^{-1} \mathrm{M}$ CR on glass substrate and c) SERS spectrum of $10^{-8} \mathrm{M}$ CR on AgT1k SERS substrate.

Also, reusability property of optimized SERS substrate was investigated for CR having $10^{-5} \mathrm{M}$ concentration. Figure 10 shows the reusability measurement, it can be seen that after washing the substrate features of the $\mathrm{CR}$ are not visible indicating washing off CR molecules from Ag NPs surface. No shift in the peak positions of CR in SERS spectra, obtained SERS enhancement factor and reusability measurements for CR molecule endorse that $\mathrm{CR}$ molecules do not chemosorb on Ag NPs and form any surface complex (See supplementary information Figure S4). Hence SERS enhancement of CR does not involve CE mechanism and only show the contribution of pure EME mechenism to its total SERS enhancement factor. In addition to EME $\mathrm{CE}$ mechanism contributes more in case of $\mathrm{MB}$ as compared to $\mathrm{CR}$ in order to get SERS enhancement factor for MB molecule up to $10^{9}$. Thus, we believe in addition to EME if the probe molecule shows CE mechanism we get higher SERS enhancement but due to chemisorption reusability od SERS substrate is limited. 


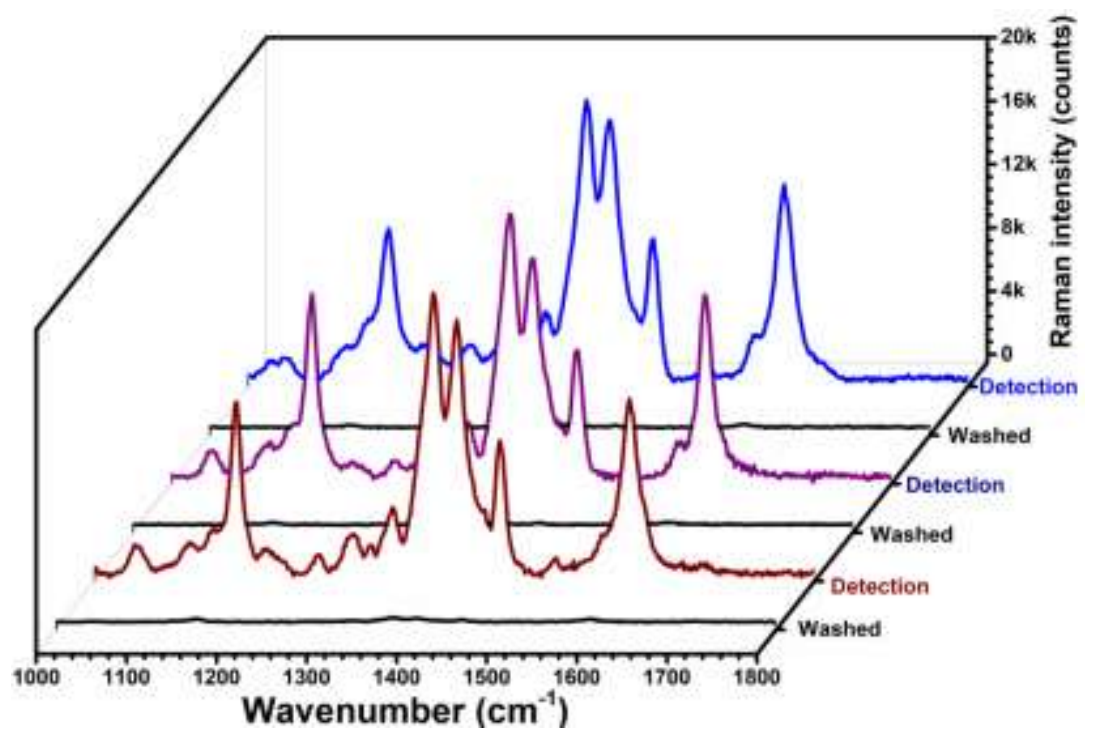

Figure 10: Reusability studies for $10^{-5} \mathrm{M}$ CR on AgT1k SERS substrate.

Further to support the claim that $\mathrm{MB}$ is chemisorbed, whereas $\mathrm{CR}$ is physisorbed on $\mathrm{Ag}$ NPs surface we have performed XPS measurements. Figure 11 shows XPS spectra of MB $\left(10^{-5}\right)$ and CR $\left(10^{-5}\right)$ adsorbed on AgT1k SERS substrate. In the deconvoluted XPS spectrum of MB adsorbed on AgT1k (Figure 11a) it can be observed that Ag 3d peak composed of two components $\mathrm{Ag}$ and $\mathrm{AgCl}$. Ag 3d5/2 gives signal of two peaks at 367.14 and $368.03 \mathrm{eV}$, whereas $\mathrm{Ag} 3 \mathrm{~d}_{3 / 2}$ gives rise to 373.33 and $374.05 \mathrm{eV}$ corresponding to $\mathrm{Ag}^{+}$of $\mathrm{AgCl}$ and $\mathrm{Ag}^{0}$, respectively [59-64]. The obtained XPS results reveal the formation of $\mathrm{Ag} @ \mathrm{AgCl}$ surface complex, confirming chemisorption of MB. On the other hand, in the XPS spectrum obtained for CR $\left(10^{-5}\right.$ M) adsorbed on AgT1k (Figure 11b) only peaks correspond to Ag0 are seen, indicating physisorption of CR. Hence SERS spectra at lower wavernumber and XPS analysis confirms that MB is chemisorbed and CR is physiosorbed on AgT1k SERS substrate. 

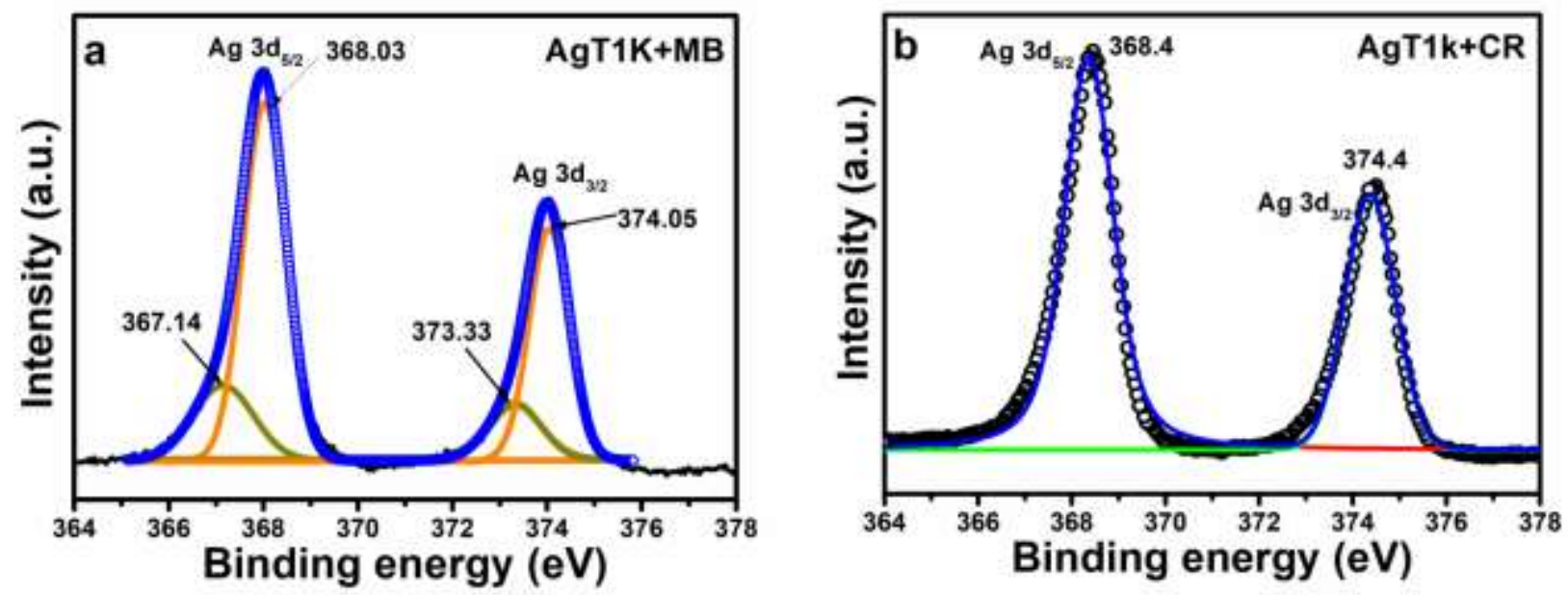

Figure 11: XPS spectra of a) MB $\left(10^{-5} \mathrm{M}\right)$ and b) CR $\left(10^{-5} \mathrm{M}\right)$ on AgT1k SERS substrate

\section{CONCLUSION}

Simple spin coating method followed by thermal reduction is successfully utilized for the uniform growth of Ag NPs. Ag NPs grown at $1000 \mathrm{rpm}$ (AgT1k) found to be optimized SERS substrate showing largest SERS activity. Sensitivity measurement shows capability of detecting trace amount of MB molecules down to $10^{-10} \mathrm{M}$ concentration. The experimentally found SERS enhancement factor $\mathrm{G}_{\mathrm{SERS}}$ values for MB $\left(\sim 3.2 \times 10^{9}\right)$ and $\mathrm{CR}\left(\sim 3.4 \times 10^{7}\right)$ are compared with the theoretically obtained ${ }^{\mathrm{em}} \mathrm{GSERS}_{\mathrm{SE}}\left(\sim 4.3 \times 10^{7}\right)$ value. For MB both EME and CE mechanisms, whereas for CR only EME mechanism contributing in SERS enhancement. This difference in enhancement mechanism lead to different trace detection limits of MB $\left(10^{-10} \mathrm{M}\right)$ and CR $\left(10^{-8}\right.$ M). Spot to spot and substrate to substrate RSDs values are found to be well below the acceptable limit (<20\%) showing good homogeneity and reproducibility of the AgT1k SERS substrate. SERS spectra at lower wavernumber and XPS analysis confirms that MB is chemisorbed on the AgT1k SERS substrate, whereas CR is not. The AgT1k SERS substrate found to be highly sensitive, reproducible and capable of detecting trace amounts of industrial pollutants, however the detection limit, GSERS values and reusability are decided by the contribution of CE SERS enhancement mechanism. 


\section{ACKNOWLEDGEMENT}

Authors are thankful to the University Grants Commission (UGC), New Delhi, India for providing Raman Microscopy facility under University with Potential for Excellence (UPE) program to Savitribai Phule Pune University, Pune, India. We are thankful to Dr. Dhananjay Bodas, Scientist, Agharkar Research Institute, Pune for technical help and fruitful discussions. We are also thankful to the Dr. Yogesh Jadhav, Department of Chemistry, Savitribai Phule Pune University, Pune for the AFM imaging.

\section{AUTHOR CONTRIBUTIONS:}

All authors contributed to the conceptualization and investigation of the study. The first draft of the manuscript was written by Imran Shaikh and all authors commented on previous versions of the manuscript. All authors read and approved the final manuscript.

\section{DECLARATIONS:}

Ethics approval: - Ethics approvals is completely considered and applied

Consent of Participate: - Consent to participate is checked

Consent for publication: - Consent for publication is checked

Conflict of interest: - The authors declare no conflict of interests

\section{Data Availability Statement}

The datasets generated during and/or analysed during the current study are available from the corresponding author on reasonable request.

\section{Funding:}

Imran Shaikh was awarded Maulana Azad National Fellowship (MANF) from University Grants Commission (UGC), New Delhi, India 


\section{REFERENCES}

1. Fleischmann, M., P.J. Hendra, and A.J. McQuillan, Raman spectra of pyridine adsorbed at a silver electrode. Chemical Physics Letters, 1974. 26(2): p. 163-166.

2. $\quad$ Etchegoin, E.L.R.P., Principles of Surface-Enhanced Raman Spectroscopy and Related Plasmonic Effects. 1 ed. 2009: Elsevier Science. 688.

3. Wang, A.X. and X. Kong, Review of Recent Progress of Plasmonic Materials and NanoStructures for Surface-Enhanced Raman Scattering. Materials (Basel), 2015. 8(6): p. 3024-3052.

4. Sharma, B., et al., SERS: Materials, applications, and the future. Materials Today, 2012. 15(1-2): p. $16-25$.

5. Fan, M., G.F. Andrade, and A.G. Brolo, A review on the fabrication of substrates for surface enhanced Raman spectroscopy and their applications in analytical chemistry. Anal Chim Acta, 2011. 693(1-2): p. 7-25.

6. Kneipp, J., H. Kneipp, and K. Kneipp, SERS--a single-molecule and nanoscale tool for bioanalytics. Chem Soc Rev, 2008. 37(5): p. 1052-60.

7. Campion, A. and P. Kambhampati, Surface-enhanced Raman scattering. Chemical Society Reviews, 1998. 27(4): p. 241.

8. $\quad$ Kneipp, K., et al., Single Molecule Detection Using Surface-Enhanced Raman Scattering (SERS). Physical Review Letters, 1997. 78(9): p. 1667-1670.

9. Hulteen, J.C., Nanosphere lithography: A materials general fabrication process for periodic particle array surfaces. Journal of Vacuum Science \& Technology A: Vacuum, Surfaces, and Films, 1995. 13(3): p. 1553.

10. Moskovits, M., Surface-enhanced Raman spectroscopy: a brief retrospective. Journal of Raman Spectroscopy, 2005. 36(6-7): p. 485-496.

11. Nie, S., Probing Single Molecules and Single Nanoparticles by Surface-Enhanced Raman Scattering. Science, 1997. 275(5303): p. 1102-1106.

12. $\mathrm{Xu}, \mathrm{H}$., et al., Electromagnetic contributions to single-molecule sensitivity in surface-enhanced Raman scattering. Physical Review E, 2000. 62(3): p. 4318-4324.

13. Otto, A., The 'chemical' (electronic) contribution to surface-enhanced Raman scattering. Journal of Raman Spectroscopy, 2005. 36(6-7): p. 497-509.

14. Willets, K.A. and R.P. Van Duyne, Localized surface plasmon resonance spectroscopy and sensing. Annu Rev Phys Chem, 2007. 58: p. 267-97.

15. Moores, A. and F.d.r. Goettmann, The plasmon band in noble metal nanoparticles: an introduction to theory and applications. New Journal of Chemistry, 2006. 30(8): p. 1121.

16. Shaikh, I.M. and S.D. Sartale, SILAR grown Ag nanoparticles as an efficient large area SERS substrate. Journal of Raman Spectroscopy, 2018. 49(8): p. 1274-1287.

17. Radziuk, D. and H. Moehwald, Prospects for plasmonic hot spots in single molecule SERS towards the chemical imaging of live cells. Physical Chemistry Chemical Physics, 2015. 17(33): p. 21072-21093.

18. Niu, Z. and Y. Fang, Surface-enhanced Raman scattering system of sample molecules in silvermodified silver film. Spectrochimica Acta Part A: Molecular and Biomolecular Spectroscopy, 2007. 66(3): p. 712-716.

19. Jiang, X., et al., Comparative study of semiconductor TiO2

and noble metal Ag substrates: The differences between chemical enhancement and electromagnetic enhancement in SERS. Journal of Raman Spectroscopy, 2018. 49(8): p. 1257-1264.

20. Kneipp, K., Chemical Contribution to SERS Enhancement: An Experimental Study on a Series of Polymethine Dyes on Silver Nanoaggregates. The Journal of Physical Chemistry C, 2016. 120(37): p. 21076-21081.

21. Iancu, S.D., et al., The role of $\mathrm{Ag}+, \mathrm{Ca} 2+, \mathrm{Pb2}+$ and Al3+ adions in the SERS turn-on effect of anionic analytes. Beilstein Journal of Nanotechnology, 2019. 10: p. 2338-2345. 
22. Leopold, N., et al., The role of adatoms in chloride-activated colloidal silver nanoparticles for surface-enhanced Raman scattering enhancement. Beilstein Journal of Nanotechnology, 2018. 9: p. 2236-2247.

23. Stefancu, A., S.D. Iancu, and N. Leopold, Selective Single Molecule SERRS of Cationic and Anionic Dyes by $\mathrm{Cl}-$ and $\mathrm{Mg} 2+$ Adions: An Old New Idea. The Journal of Physical Chemistry C, 2021. 125(23): p. 12802-12810.

24. Cheung, W.H., Y.S. Szeto, and G. McKay, Enhancing the adsorption capacities of acid dyes by chitosan nano particles. Bioresource Technology, 2009. 100(3): p. 1143-1148.

25. Hou, X., et al., Periodic silver nanocluster arrays over large-area silica nanosphere template as highly sensitive SERS substrate. Applied Surface Science, 2018. 437: p. 92-97.

26. D'Andrea, C., et al., Decoration of silicon nanowires with silver nanoparticles for ultrasensitive surface enhanced Raman scattering. Nanotechnology, 2016. 27(37): p. 375603.

27. Tao, W., et al., Periodic silver nanodishes as sensitive and reproducible surface-enhanced Raman scattering substrates. RSC Adv., 2014. 4(7): p. 3487-3493.

28. Camelio, S., et al., Optimization of growth and ordering of Ag nanoparticle arrays on ripple patterned alumina surfaces for strong plasmonic coupling. Nanotechnology, 2014. 25(3): p. 035706.

29. Theiss, J., et al., Plasmonic Nanoparticle Arrays with Nanometer Separation for HighPerformance SERS Substrates. Nano Letters, 2010. 10(8): p. 2749-2754.

30. Song, Y., et al., The electrodeposition of Ag nanoparticles on a type I collagen-modified glassy carbon electrode and their applications as a hydrogen peroxide sensor. Nanotechnology, 2009. 20(10): p. 105501.

31. Abu Hatab, N.A., J.M. Oran, and M.J. Sepaniak, Surface-Enhanced Raman Spectroscopy Substrates Created via Electron Beam Lithography and Nanotransfer Printing. ACS Nano, 2008. 2(2): p. 377-385.

32. Ansari, A.A. and S.D. Sartale, Narrow size distributed Ag nanoparticles grown by spin coating and thermal reduction: effect of processing parameters. Materials Research Express, 2016. 3(8): p. 085023.

33. Ansari, A.A. and S.D. Sartale, Effect of processing parameters on size, density and oxygen reduction reaction $(O R R)$ activity of $P d$ nanoparticles grown by spin coating. Surface and Coatings Technology, 2015. 281: p. 68-75.

34. Ansari, A.A. and S.D. Sartale, Controlled growth of thermally stable uniform-sized Ag nanoparticles on flat support and their electrochemical activity. Applied Physics A, 2015. 119(2): p. 503-516.

35. Kuipers, E.W., C. Laszlo, and W. Wieldraaijer, Deposition of nanocrystals on flat supports by spin-coating. Catalysis Letters, 1993. 17(1-2): p. 71-79.

36. Gücek, A., et al., Adsorption and kinetic studies of cationic and anionic dyes on pyrophyllite from aqueous solutions. Journal of Colloid and Interface Science, 2005. 286(1): p. 53-60.

37. Dawood, S. and T.K. Sen, Removal of anionic dye Congo red from aqueous solution by raw pine and acid-treated pine cone powder as adsorbent: Equilibrium, thermodynamic, kinetics, mechanism and process design. Water Research, 2012. 46(6): p. 1933-1946.

38. Sartale, S.D. and A.A. Ansari, Growth of Ag Nanoparticles by Spin Coating. Journal of Nano Research, 2013. 24: p. 163-167.

39. Cavallini, M., et al., Micro- and nanopatterning by lithographically controlled wetting. Nature Protocols, 2012. 7(9): p. 1668-1676.

40. Mirji, S.A., et al., Adsorption of methanol on Si(100)/SiO2 and mesoporous SBA-15. Colloids and Surfaces A: Physicochemical and Engineering Aspects, 2006. 287(1-3): p. 51-58.

41. Doornkamp, C., et al., Exploration of the deposition of submicrometer particles by spin-coating. Journal of Materials Research, 2011. 10(2): p. 411-424. 
42. Partridge, A., S.L.G. Toussaint, and C.F.J. Flipse, An AFM investigation of the deposition of nanometer-sized rhodium and copper clusters by spin coating. Applied Surface Science, 1996. 103(2): p. 127-140.

43. Xiao, G.N. and S.Q. Man, Surface-enhanced Raman scattering of methylene blue adsorbed on cap-shaped Silver nanoparticles. Chemical Physics Letters, 2007. 447(4-6): p. 305-309.

44. Li, C., et al., Analysis of trace methylene blue in fish muscles using ultra-sensitive surfaceenhanced Raman spectroscopy. Food Control, 2016. 65: p. 99-105.

45. Naujok, R.R., R.V. Duevel, and R.M. Corn, Fluorescence and Fourier Transform surfaceenhanced Raman scattering measurements of methylene blue adsorbed onto a sulfur-modified gold electrode. Langmuir, 1993. 9(7): p. 1771-1774.

46. Xiao, G.-N. and S.-Q. Man, Surface-enhanced Raman scattering of methylene blue adsorbed on cap-shaped silver nanoparticles. Chemical Physics Letters, 2007. 447(4-6): p. 305-309.

47. Bottger, G.L. and C.V. Damsgard, Second order Raman spectra of AgCl and AgBr crystals. Solid State Communications, 1971. 9(15): p. 1277-1280.

48. Niemantsverdriet, J.W., et al., Realistic surface science models of industrial catalysts. Applied Surface Science, 1999. 144-145: p. 366-374.

49. Fierro, S. and C. Comninellis, Kinetic study of formic acid oxidation on Ti/IrO2 electrodes prepared using the spin coating deposition technique. Electrochimica Acta, 2010. 55(23): p. 7067-7073.

50. Johnson, P.B. and R.W. Christy, Optical Constants of the Noble Metals. Physical Review B, 1972. 6(12): p. 4370-4379.

51. Zhang, J., et al., Surface-enhanced Raman scattering properties of multi-walled carbon nanotubes arrays-Ag nanoparticles. Carbon, 2016. 100: p. 395-407.

52. Liu, Y., et al., Localized and propagating surface plasmon co-enhanced Raman spectroscopy based on evanescent field excitation. Chemical Communications, 2011. 47(13): p. 3784.

53. Doering, W.E. and S. Nie, Single-Molecule and Single-Nanoparticle SERS: Examining the Roles of Surface Active Sites and Chemical Enhancement. The Journal of Physical Chemistry B, 2002. 106(2): p. 311-317.

54. W. Gao, J.T.X., C. Cheng, S. Qiu, S.X. Jiang, Rapid and highly sensitive SERS detection of fungicide based on flexible "wash free" metallic textile. Applied Surface Science 2020. 512: p. 144693.

55. Natan, M.J., Concluding Remarks : Surface enhanced Raman scattering. Faraday Discussions, 2006. 132: p. 321.

56. Fu, Q., et al., Highly Reproducible and Sensitive SERS Substrates with Ag Inter-Nanoparticle Gaps of $5 \mathrm{~nm}$ Fabricated by Ultrathin Aluminum Mask Technique. ACS Applied Materials \& Interfaces, 2015. 7(24): p. 13322-13328.

57. Hiramatsu, H. and T. Saito, Vertical flow apparatus for enhancement and efficient collection of Raman signal. Journal of Raman Spectroscopy, 2014. 45(2): p. 208-210.

58. Pigorsch, E., A. Elhaddaoui, and S. Turrell, Spectroscopic study of pH and solvent effects on the structure of Congo red and its binding mechanism to amyloid-like proteins. Spectrochimica Acta Part A: Molecular Spectroscopy, 1994. 50(12): p. 2145-2152.

59. Changcun Han, L.G., Changfeng Chen,Yujing Li, Zhen Zhao, Xinlai Xiao, Zhiliang Lib and Junlong Zhangb, Site-selected synthesis of novel Ag@AgCl nanoframes with efficient visible light induced photocatalytic activity. Journal of Materials Chemistry A, 2012. 22: p. 13153.

60. Fan, Y.Y., et al., Controllable synthesis of coloured $\mathrm{AgO} / \mathrm{AgCl}$ with spectral analysis for photocatalysis. Rsc Advances, 2018. 8(44): p. 24812-24818.

61. Liqun Ye, J.L., Chuqing Gong, Lihong Tian, Tianyou Peng, and Ling Zan, Two Different Roles of Metallic Ag on Ag/AgX/BiOX $(X=C l$, Br) Visible Light Photocatalysts: Surface Plasmon Resonance and Z- Scheme Bridge. ACS catalyst. 
62. Tang, Y.X., et al., In situ formation of large-scale Ag/AgCl nanoparticles on layered titanate honeycomb by gas phase reaction for visible light degradation of phenol solution. Applied Catalysis B-Environmental, 2011. 106(3-4): p. 577-585.

63. Dong, Y.Y., et al., Synthesis and characterization of Ag@ AgCl-reinforced cellulose composites with enhanced antibacterial and photocatalytic degradation properties. Scientific Reports, 2021. 11(1).

64. An, C.H., et al., Synthesis of $\mathrm{Ag} @ \mathrm{AgBr} / \mathrm{AgCl}$ heterostructured nanocashews with enhanced photocatalytic performance via anion exchange. Journal of Materials Chemistry, 2012. 22(26): p. 13153-13158. 